University of Nebraska - Lincoln

DigitalCommons@University of Nebraska - Lincoln

USDA National Wildlife Research Center - Staff Publications
U.S. Department of Agriculture: Animal and Plant Health Inspection Service

2013

Potential economic benefits of eliminating canine rabies

Stephanie A. Shwiff

USDA/APHIS/WS National Wildlife Research Center, stephanie.a.shwiff@aphis.usda.gov

Katie Hampson

University of Glasgow

Aaron M. Anderson

USDA/APHIS/WS National Wildlife Research Center, aaron.m.anderson@aphis.usda.gov

Follow this and additional works at: https://digitalcommons.unl.edu/icwdm_usdanwrc

Shwiff, Stephanie A.; Hampson, Katie; and Anderson, Aaron M., "Potential economic benefits of eliminating canine rabies" (2013). USDA National Wildlife Research Center - Staff Publications. 1243. https://digitalcommons.unl.edu/icwdm_usdanwrc/1243

This Article is brought to you for free and open access by the U.S. Department of Agriculture: Animal and Plant Health Inspection Service at DigitalCommons@University of Nebraska - Lincoln. It has been accepted for inclusion in USDA National Wildlife Research Center - Staff Publications by an authorized administrator of DigitalCommons@University of Nebraska - Lincoln. 
Review

\title{
Potential economic benefits of eliminating canine rabies
}

\author{
Stephanie Shwiff ${ }^{\text {a,* }}$, Katie Hampson ${ }^{\mathrm{b}}$, Aaron Anderson ${ }^{\mathrm{a}}$ \\ ${ }^{a}$ USDA/APHIS/WS National Wildlife Research Center, 4101 LaPorte Ave. Fort Collins, CO 80521, USA \\ ${ }^{\mathrm{b}}$ Boyd Orr Centre for Population and Ecosystem Health, Graham Kerr Building Medical, Veterinary E Life Sciences, University of Glasgow, Glasgow G12 8QQ United Kingdom
}

\section{A R T I C L E I N F O}

\section{Article history:}

Received 16 November 2012

Revised 22 February 2013

Accepted 1 March 2013

Available online 13 March 2013

\section{Keywords:}

Rabies

Canine rabies

Postexposure prophylaxis

Zoonosis

Economic analysis

\begin{abstract}
A B S T R A C T
Although canine rabies has been eliminated from industrialized countries, infected dogs remain the primary source of human and livestock exposures in Asia, Africa and much of South America. Human deaths are the most important direct economic impact of canine rabies, followed by livestock losses and the cost of PEP, while expenses associated with dog vaccination and control are major indirect impacts. The global burden of rabies disproportionately affects Asia, which experiences more than half of human rabies deaths and approximately $65 \%$ of livestock losses, and performs more than $90 \%$ of postexposure prophylaxis (PEP). Africa is second to Asia in terms of human deaths and livestock losses, but administers the least number of PEPs of the three regions. Recent experience in Latin America shows that efforts to reduce human deaths from rabies through expanded dog vaccination and improved access to PEP result in significant monetary savings. The elimination of canine rabies would lead to major economic benefits in developing countries that are often the least capable of dealing with the disease. This article forms part of a symposium in Antiviral Research on the elimination of canine rabies.
\end{abstract}

Published by Elsevier B.V.

\section{Contents}

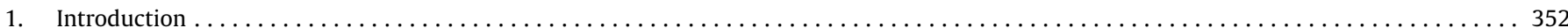

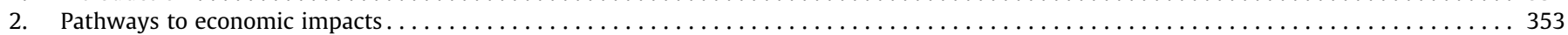

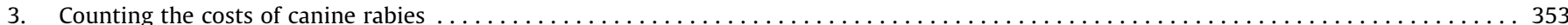

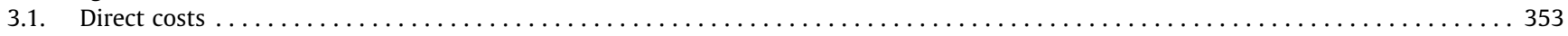

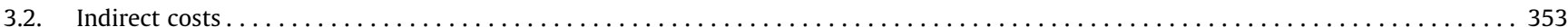

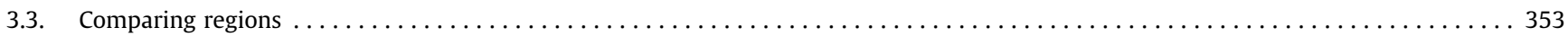

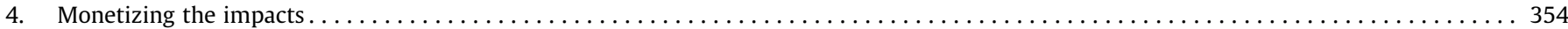

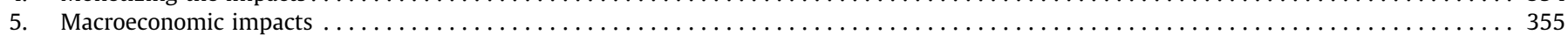

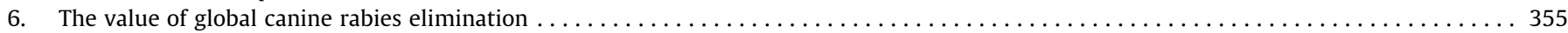

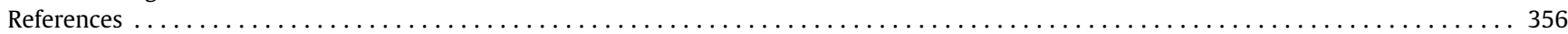

\section{Introduction}

Although canine rabies has been eliminated from industrialized countries, dogs remain the primary source of human and livestock exposure in Asia, Africa and much of Latin America (Meslin and Briggs, 2013). Canine rabies is an economically unique zoonosis because most of its associated costs do not result from illness, but are the consequence of human deaths and efforts

\footnotetext{
* Corresponding author. Tel.: +1 970266 6150; fax: +1 9702666089 .

E-mail addresses: Stephanie.A.Shwiff@aphis.usda.gov, emmashwiff@gmail.com (S. Shwiff).
}

to prevent the disease in humans, livestock and companion animals. This unique pattern of costs reflects two basic facts: the case fatality rate of rabies is nearly $100 \%$, and the disease is completely preventable through timely postexposure prophylaxis (PEP) with rabies vaccine (Blanton et al., 2010). As a result, many individuals who are at very low risk of developing the disease still seek postexposure vaccination, regardless of the recommendation of health professionals (Rupprecht et al., 2010). Although the close relationship between humans and dogs is the primary cause of rabies exposures, that relationship also provides many opportunities for mitigating its impact.

Rabies exposures in humans or livestock result in economic impacts associated with vaccination or death. Because rabies 
patients die quickly, and there is no effective therapy, the cost of illness is relatively small, especially in the developing world. In contrast, the major direct costs arising from factors such as PEP and livestock deaths have been characterized in numerous studies (Shwiff et al., 2007; Sterner et al., 2009; Zinsstag et al., 2009). Canine rabies also has indirect costs, including vaccination of livestock and companion animals and testing of dogs suspected of rabies (Anderson et al., 2012; Narrod et al., 2012). Other impacts of rabies on the broader economy can be captured by examining changes in different sectors that result from the direct and indirect impacts of the disease (Diao et al., 2009). Knobel et al. (2005) estimated global monetary expenses resulting from rabies at $\$ 695$ million annually. Given the significant economic burden of canine rabies, there are substantial benefits to be derived from its elimination.

A number of countries have succeeded in eliminating canine rabies, resulting in long-term cost savings (Rupprecht et al. 2008; Schneider et al. 2007; Shwiff et al., 2008). Eliminating canine rabies requires an integrated, cooperative approach, in which public and private human and animal health professionals work together (Taylor and Partners for Rabies Prevention, 2013). This "One Health" strategy achieves benefits that could not be attained by either group working alone (Schwabe, 1984). This approach was useful recently in eliminating canine rabies from Texas, through the coordinated efforts of state and federal agencies in an oral rabies vaccination program (Shwiff et al., 2008). An understanding of the economic benefits that could be achieved through the elimination of canine rabies in a broader context (i.e., in Africa or Asia) is central to promoting cooperation between health sectors and ensuring that management resources are allocated efficiently.

\section{Pathways to economic impacts}

Like all zoonotic diseases, rabies is maintained in an animal reservoir. In developed countries, where canine rabies has been eliminated, the virus may continue to circulate in wildlife, whereas in most developing countries the principal reservoir is domestic dogs. Human and livestock exposure is based on a multitude of risk factors (Zinsstag et al., 2007; Narrod et al., 2012), and there are several pathways by which canine rabies causes economic damage (Fig. 1). Dog vaccination status is of paramount importance and while it represents a cost (indicated by the shaded box), it minimizes or eliminates further economic impact. In contrast, unvaccinated dogs, or dogs whose vaccination status is unknown, represent a pathway to further economic impacts. If the vaccination status of a dog can be readily determined, the initial cost of vaccination negates any further downstream impacts, but when humans and livestock are exposed to a dog that might have rabies, or whose vaccination status is unknown, further costs may be incurred. Livestock vaccination also represents an up-front cost, without further impacts, while unvaccinated, exposed animals will either not become infected, or will die from rabies. Similarly, there are two pathways following human exposure: the individual either seeks medical treatment and is given PEP, incurring direct and indirect costs (Shwiff et al., 2008), or he does not receive PEP, and either remains well or dies from rabies, leading to further costs.

Macroeconomic impacts of human and livestock exposure to rabid dogs can also be significant. For example, when livestock die from rabies, the producer's income decreases, resulting in a drop in consumer spending, which in turn leads to reduced demand across many sectors of the economy, causing prices to fall and sales to decline. Ultimately, this results in less demand for labor and lower total production of goods and services.

\section{Counting the costs of canine rabies}

\subsection{Direct costs}

The direct costs of canine rabies can be divided into three categories: human deaths, PEP and livestock losses. Although human infections are rare in the developed world, they constitute the greatest direct economic impact of canine rabies in many countries in Africa and Asia. Adjusting estimates from Knobel et al. (2005) to account for population growth yields a current estimate of over 69,000 rabies deaths per year in Africa and Asia. Canine rabies still occurs in the Western Hemisphere; the Global Alliance for Rabies Control (2012) reports that, in 2008-11, an average of 15 people died each year from canine rabies in Latin America.

Other direct impacts of canine rabies include the cost of PEP and livestock losses. Adjusting the 2004 estimates from Knobel et al. (2005) to account for population growth, PEP was administered nearly 9 million times in Asia and more than 260,000 times in Africa (Table 1). Even though the incidence of canine rabies in Latin America has fallen dramatically in recent decades, the perceived risk remains, and it is likely that PEP use remains elevated; during 1990-2003, nearly one million people received PEP each year. During the same time period, dogs accounted for $65 \%$ of potential exposures based on the information obtained on the attack animal, yielding an annual estimate of more than 600,000 PEP cases (Schneider et al., 2007). In terms of livestock deaths (Table 1), Asia is most heavily burdened, with an estimated annual loss of some 21,000 cattle, while approximately half that number die in Africa, and losses in Latin American are negligible (Knobel et al., 2005). Lack of data prevented estimation of the losses of other types of livestock, but it likely that such losses follow patterns similar to those estimated for cattle.

\subsection{Indirect costs}

Diagnostic testing and observation and dog vaccination constitute the two main indirect costs of canine rabies. Additionally, dog population control is used in some regions and is a relevant cost. Following a possible human exposure to a rabid dog, an animal control agency will attempt to obtain the dog and determine its rabies status. Such testing and observation plays an important role in disease surveillance. Approximately 16,500 diagnostic tests are performed annually in Asia and 5300 in Africa (Knobel et al., 2005). In Latin American, by contrast, more than 73,000 tests were performed each year from 1990 to 2003 (Schneider et al., 2007).

Vaccination is a crucial component of rabies control, because if a dog is known to have been vaccinated, then its bite has no further economic impact related to rabies (Fig. 1). Although common in developed countries, knowledge of a dog's vaccination status is unusual in Africa and Asia, due to the inability to establish ownership. A number of countries in Latin America have carried out large-scale campaigns, resulting in the annual vaccination of some 42 million dogs based on data collected from 2001-2003 (Table 2) (Schneider et al., 2007). Asia has lagged slightly behind, with approximately 40 million immunizations, while Africa vaccinated only 6.7 million dogs (Knobel et al., 2005).

\subsection{Comparing regions}

The burden of canine rabies falls most heavily on Asia (e.g., China and India), which experiences over half of human and cattle deaths, and performs more than 90\% of PEP administrations and just under half of dog vaccinations. Total human and livestock deaths in Latin America are much lower than in Africa and Asia, probably as a direct result of coordinated efforts by many countries 


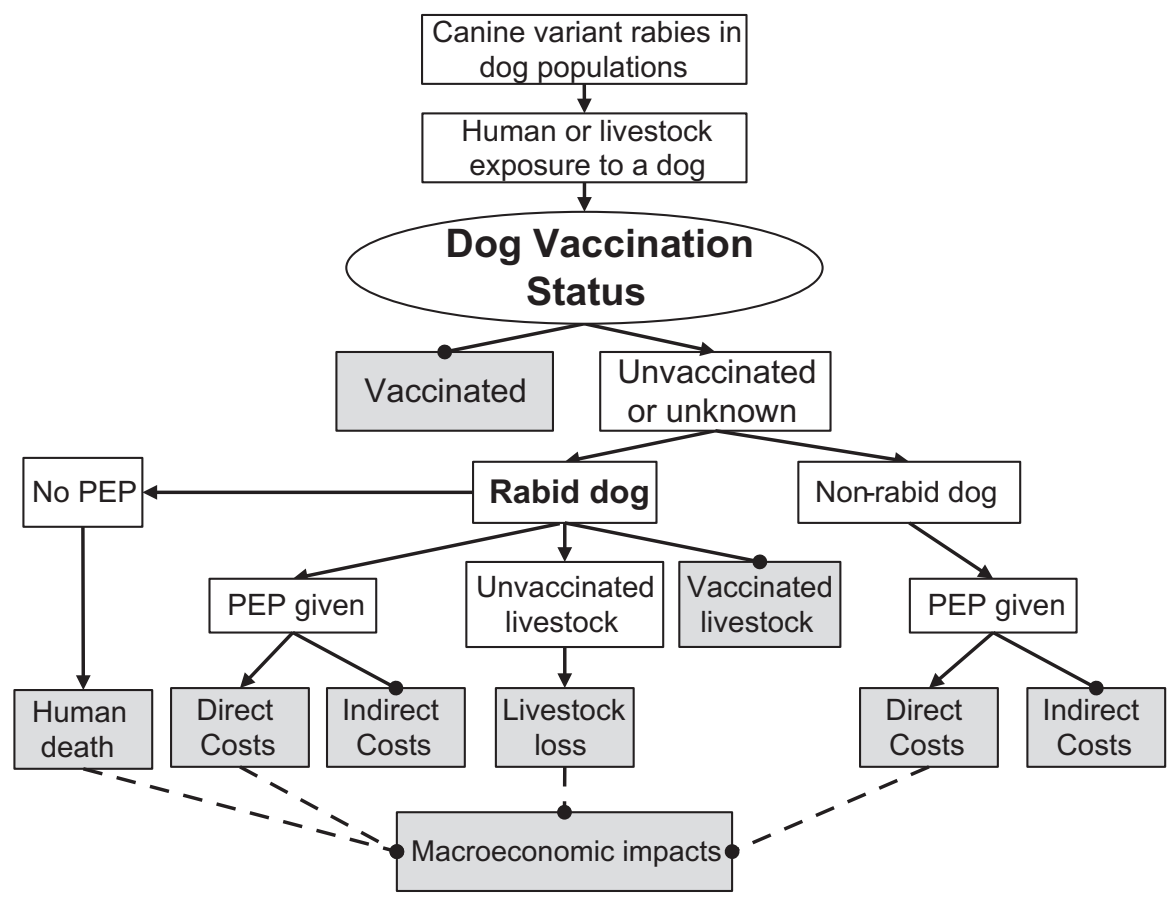

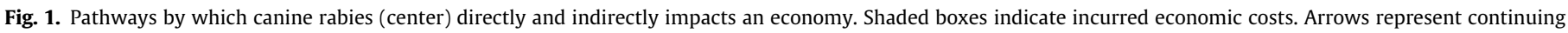
pathways to measurable economic impacts; dots represent the end of a pathway.

\section{Table 1}

Annual direct impacts of canine rabies in terms of human and cattle deaths and PEP cases (in thousands). The global burden of rabies disproportionately affects Asia, which experiences more than half of human rabies deaths and approximately $65 \%$ of livestock losses, and performs more than $90 \%$ of PEP.

\begin{tabular}{lllll}
\hline & Latin America & Africa & Asia & Total \\
\hline Human deaths & .002 & 31 & 38 & 69 \\
PEP cases & 620 & 260 & 8,700 & 9000 \\
Cattle deaths & .002 & 11 & 21 & 32 \\
\hline
\end{tabular}

in the region to eliminate canine rabies (Schneider et al., 2007), which also explains the high annual number of dog vaccinations. Expenditures to fight rabies in Africa are much lower than in Latin America and Asia, but many deaths could be prevented through better access to PEP and expanded dog vaccination efforts.

The most dramatic difference between Asia, Africa and Latin America is seen in access to PEP. For each fatal case of human rabies, Latin America performs over 41,000 PEPs, while Asia and Africa give around 200 and 8, respectively. Greater access to PEP in Latin America clearly decreases the number of human lives lost. The three regions show a similar disparity in dog vaccination rates. In Latin America, over 2.8 million dogs were vaccinated per human life lost, while only some 1000 were vaccinated in Asia and 200 in Africa. The large number of dog vaccinations in Latin America has had an obvious impact on the number of humans who die from rabies in the region. Cattle losses show a similar trend: for each cow

\section{Table 2}

Annual indirect impacts of canine rabies measured by the number of animal tests and dog vaccinations (in thousands). A number of countries in Latin America have carried out large-scale vaccination campaigns, which have had a large impact on the number of humans who die from rabies in the region.

\begin{tabular}{lllll}
\hline & Latin America & Africa & Asia & Total \\
\hline Animal tests & 74 & 5.3 & 16.5 & 96 \\
Dog vaccines & 42,000 & 6,700 & 40,000 & 89,000 \\
\hline
\end{tabular}

lost to rabies in Latin America, over 1.7 million dogs were vaccinated, while in Asia and Africa the numbers were approximately 1900 and 600, respectively.

\section{Monetizing the impacts}

Examining the direct and indirect costs of canine rabies shown in Tables 1 and 2 further emphasizes regional differences. Human deaths have the greatest economic impact. As the estimated value of a statistical life (VSL) ranges from around $\$ 1$ million to over $\$ 12$ million (Bellavance et al., 2009), the total annual global cost of deaths from canine rabies is in the tens of billions of dollars, dwarfing the impact of all other variables combined. There is considerable variation in VSL estimates, but a number of extensive meta-analyses have been performed. By correcting for publication selectivity bias, Doucouliagos et al. (2012) arrive at an estimate of $\$ 2.2$ million for total cost of human deaths due to rabies. Consideration of the effects of including workers' compensation, time trends, and workers' average income yields an even lower estimate of $\$ 1.8$ million. We assumed a VSL of $\$ 1.8$ million because this was the most recent and thorough estimate available from a meta-analysis, and VSL estimates from developing countries are often lower than those from developed countries (Bowland and Beghin, 2001; Hoffman et al., 2012).

PEP is the second most expensive element in Table 1 Pep has been estimated to cost $\$ 30-40$ per application across all regions, including different types of vaccines, material and overhead (Knobel et al., 2005; Schneider et al., 2007). The expected PEP costs were calculated as the probability-weighted summation of the various costs and account for all injections and visits. We assumed all patients receive tissue culture vaccine on the grounds that use of nerve tissue vaccine is minimal and decreasing. Although the probability of patients receiving rabies immunoglobulin (RIG) is small we based our estimates of the use of either the human RIG (hRIG) or equine RIG (eRIG) in all regions on published estimates (Knobel et al., 2005). The percentage of patients receiving intradermal or intramuscular vaccine as well as the number of doses, cost of each 
dose and the number of visits was also based on published information (Knobel et al., 2005).

The significantly larger number of PEP administrations in Asia (Table 3 ) results in greater costs. Although more animal tests are performed in Latin America, the total cost is only a fraction of PEP, resulting in an insignificant overall impact. The numbers and cost of dog vaccination are roughly equal in Latin America and Asia, yielding similar economic impacts. Lastly, a greater number of cattle deaths and higher values per animal result in higher livestock losses in Asia than other regions. The cost of rabies in Latin America, Africa, and Asia that is associated with the impacts listed in Table 3 total $\$ 530$ million.

As shown in Table 3, Asia accrues most of the global economic impact of canine rabies, excluding human deaths, while Africa incurs the lowest costs. The fact that Latin America has few cases of disease, but large expenditures for PEP, animal testing and dog vaccination, shows that it may take years for a fall in rabies prevalence to result in reduced expenditures for treatment, vaccination and surveillance, because the public's perceived risk of the disease remains elevated.

Regional differences in the allocation of resources to each category highlight the diversity of rabies elimination efforts (Table 4). In Latin American, the majority of resource allocation is to dog vaccination which ultimately prevents human exposure to rabid dogs. The second largest regional allocation toward rabies elimination is to treatment of possible human infection through PEP, thereby reducing human fatality. This further supports that this region has progressed farther toward rabies elimination than the other regions reflecting the fact that the majority of resources are allocated to prevention as opposed to treatment. Asia is the opposite in that even though the total number of dog vaccinations from Table 2 was similar to the number in Latin America, actual within-region resources are allocated significantly more to human treatment. A reallocation of resources within Asia from treatment to prevention (e.g., dog vaccination) could likely reduce overall costs by reducing the need for PEP. In Africa, the allocation of resources is relatively evenly split between prevention (dog vaccination) and treatment (PEP), although given the paucity of overall resource allocation to fight rabies it is difficult to make an interpretation of this result.

\section{Macroeconomic impacts}

Human and livestock deaths from rabies and the cost of PEP produce regional macroeconomic impacts, including changes in

\section{Table 3}

Percentage of total global costs of canine rabies (excluding human death) by category and region. Asia accrues most of the global economic impact of canine rabies, while Africa incurs the lowest costs. We estimate that these costs total $\$ 530$ million.

\begin{tabular}{lclrr}
\hline Category & Latin America & Africa & Asia & Total \\
\hline PEP cases & 4.5 & 1.9 & 64.7 & 71.1 \\
Animal tests & 0.1 & 0.0 & 0.0 & 0.1 \\
Dog vaccines & 12.4 & 2.0 & 11.7 & 26.1 \\
Cattle deaths & 0.0 & 0.4 & 2.4 & 2.8 \\
Total & 17.0 & 4.3 & 78.8 & \\
\hline
\end{tabular}

Table 4

Percentage of total canine rabies allocation (excluding human death) within each region by category.

\begin{tabular}{lcrr}
\hline Category & Latin America & Africa & \multicolumn{1}{c}{ Asia } \\
\hline PEP cases & 26.54 & 44.39 & 82.07 \\
Animal tests & 0.56 & 0.16 & 0.03 \\
Dog vaccines & 72.9 & 46.28 & 14.88 \\
Cattle deaths & 0.01 & 9.17 & 3.03 \\
\hline
\end{tabular}

income and employment, which arise from multiple sources. First, human death and loss of income while seeking PEP reduce consumer spending throughout the economy. Canine rabies also causes significant livestock losses, which reduce producers' incomes, resulting in a fall in spending in other sectors of the local and regional economy. These macroeconomic impacts illustrate how rabies affects persons who are not at direct risk of the disease. In this regard, Regional Economic Analysis (REA) allows for the estimation of disease impacts in terms such as income and employment, which are important to the general public. Although models such as IMPLAN (Impact Analysis for Planning, Minnesota IMPLAN $^{\circledR}$ Group) and REMI (Regional Economic Modeling Inc.) are commonly used in REA, a lack of data often prevents their application in developing countries (MIG Inc., 2012; Treyz et al., 1991).

\section{The value of global canine rabies elimination}

The composition of the economic impacts of canine rabies in Latin America, Africa and Asia reveals something about the effectiveness of management strategies within each region. In Latin America, the distribution of costs reflects significant efforts to eliminate the disease, because human and livestock losses are low, but expenses for PEP and dog vaccination are relatively high. This may also reflect a lag time between investing in canine rabies reduction and the return on that investment, in terms of reduced PEP and fewer vaccinations. As noted above, even when the prevalence of canine rabies is reduced, it may take some time before individuals perceive their risk to be lower, and become less likely to seek PEP inappropriately, after incidents unlikely to constitute rabies exposure.

In contrast to Latin America, Africa experiences the world's second highest total costs from human and livestock deaths from rabies, while relatively little overall effort is devoted to PEP, animal tests and dog vaccinations. This pattern of economic impacts indicates that Africa is the region furthest from eliminating canine rabies, but it also implies that large benefits could be derived by shifting costs towards prevention.

In Asia, even though significant investments have been made in PEP, dog vaccination, human deaths and livestock losses remain high, indicating that prevention efforts still fall significantly short of the levels needed to drive costs down. This suggests that prevention efforts are incomplete, because while PEP and dog vaccination are crucial for preventing rabies in humans and livestock, animal testing represents the surveillance portion of prevention efforts. Without adequate surveillance, it may be difficult to gauge the disease status of dog populations, resulting in incorrect estimates of the level of vaccination needed.

Because the economic costs of canine rabies can be seen as benefits that would be realized if it were eliminated, it is essential to understand the pathways to economic impacts of the disease. Importantly, the global burden of canine rabies is not distributed equally, and it disproportionately affects regions with limited resources, that are least capable of responding to the disease. Latin America, Africa and Asia are at different stages of canine rabies elimination. Due to the nature of the reservoir and the close relationship between dogs and humans, dog vaccination is a crucial component in all regions. In Latin America vaccination campaigns are one of the primary factors that have reduced human death, while in Africa the lack of dog vaccination is one of the main factors behind the high human death rate. The benefits of eliminating the disease extend beyond persons directly exposed to rabid dogs, to everyone in the community. Understanding the economic impact of canine rabies and the benefits that can be achieved by its elimination is central to the effective cooperation of health sectors. 


\section{References}

Anderson, A., Shwiff, S., Gebhardt, K., Ramírez, A. J., Shwiff, S., Kohler, D., Lecuona, L., 2012. Economic evaluation of vampire bat (Desmodus rotundus) rabies prevention in Mexico. Transbound. Emerg. Dis. doi: 10.1111/tbed.12007.

Bellavance, F., Dionne, G., Lebeau, M., 2009. The value of a statistical life: a metaanalysis with a mixed effects regression model. J. Health Econ. 28, 444464.

Blanton, J.D., Palmer, D., Rupprecht, C.E., 2010. Rabies surveillance in the United States during 2009. J. Am. Vet. Med. Assoc. 237 (6), 646-657.

Bowland, B.J., Beghin, J.C., 2001. Robust estimates of value of statistical life for developing economics. J. Policy Model. 23, 385-396.

Diao, X., Alpuerto, V., Nwafo, M., 2009. Economy wide impact of Avian Flu in Nigeria - a dynamic CGE model analysis. HPAI Research Brief No. 15 <www.ifpri.org/ sites/files/publications/hpairb15.pdf>.

Doucouliagos, C., Stanley, T.D., Giles, M., 2012. Are estimates of the value of a statistical life exaggerated? J. Health Econ. 31, 197-206.

Global Alliance for Rabies Control, 2012. International Collaboration for Canine Rabies Elimination in Latin America. <http://rabiescontrol.net/news/newsarchive/international-collaboration-for-canine-rabies-elimination-in-latinamerica.html> (accessed 09.13.12.)

Hoffman, S., Qin, P., Krupnick, A., Badrakh, B., Batbaatar, S., Altangerel, E., Sereeter, L., 2012. The willingness to pay for mortality risk reductions in Mongolia. Resour. Energy Econ. 34, 493-513.

Knobel, D.L., Cleaveland, S., Coleman, P.G., Fevre, E.M., Meltzer, M.I., Miranda, M.E.G., Shaw, A., Zinsstag, J., Meslin, F., 2005. Re-evaluating the burden of rabies in Africa and Asia. Bull. World Health Organ. 83 (5), 360-368.

Meslin, F.X., Briggs, D., in press. Eliminating canine rabies, the principal source of human infection: what will it take? Antiviral Res.

MIG Inc., 2012. Economic impact analysis. <www.implan.com> (accessed Feb 2013).

Narrod, C., Zinsstag, J., Tiongco, M., 2012. A one health framework for estimating the economic costs of zoonotic disease on society. EcoHealth 9, 150-162.

Rupprecht, C.E. et al., 2008. Can Rabies be eradicated? Dev Biol 131, 95-121.
Rupprecht, C.E. et al, 2010. Use of a reduced (4-dose) vaccine schedule for postexposure prophylaxis to prevent human rabies: recommendations of the advisory committee on immunization practices. M.M.W.R. Recomm. Rep. 59 (RR-2), 1-9.

Schneider, M.C., Belotto, A., Ade, M.P., Hendrickx, S., Leanes, L.F., Rodrigues, M.J., Medina, G., Correa, E., 2007. Current status of human rabies transmitted by dogs in Latin America. Cad Saude Publica 23 (9), 2049-2063.

Schwabe, C.W., 1984. Veterinary Medicine and Human Health. Williams \& Wilkins, Baltimore, pp. 1-680.

Shwiff, S.A., Sterner, R.T., Jay-Russell, M., Parikh, S., Bellomy, A., 2007. Direct and indirect costs of rabies exposure: a retrospective study in Southern California (1998-2002). J. Wildl. Dis. 43 (2), 251-257.

Shwiff, S.A., Kirkpatrick, K.N., Sterner, R.T., 2008. Economic evaluation of a Texas oral rabies vaccination program for control of a domestic dog-coyote rabies epizootic: 1995-2006. J. Am. Vet. Med. Assoc. 233, 1736-1741.

Shwiff, S., Aenishaenslin, C., Ludwig, A., Berthiaume, P., Bigras-Poulin, M., Kirkpatrick, K., Lambert, L., Bélanger, D., in press. Bioeconomic modelling of raccoon rabies spread management impacts in Quebec, Canada. Transbound. Emerg. Dis. doi: 10.1111/j.1865-1682.2012.01351.

Sterner, R.T., Meltzer, M.I., Shwiff, S.A., Slate, D., 2009. Tactics and economics of wildlife oral rabies vaccination, Canada and the United States. Emerg. Infect. Dis. $15,1176-1184$

Taylor, L., the Partners for Rabies Prevention, in press. Eliminating canine rabies: the role of public-private partnerships. Antiviral Res.

Treyz, G.I., Rickman, D.S., Shao, G., 1991. The REMI economic-demographic forecasting and simulation model. Int. Reg. Sci. Rev. 14 (3), 221-253.

Zinsstag, J., Schelling, E., Roth, F., Bonfoh, B., de Savigny, D., Tanner, M., 2007. Human benefits of animal interventions for zoonosis control. Emerg. Infect. Dis. 13 (4), 527-531.

Zinsstag, J., Durr, S., Penny, M.A., Mindekem, R., Roth, F., Menendez Gonzalez, S., Naissengar, S., Hattendorf, J., 2009. Transmission dynamics and economics of rabies control in dogs and humans in Africa City. Proc. Natl. Acad. Sci. 106 14996-15001. 